\title{
Applying Resonant Electromagnetic Fields in Membrane Channels of Tumor Cells: The Future of Cancer Treatment
}

\author{
Emanuele Calabrò ${ }^{1,5 *}$ and Salvatore Magazù ${ }^{1-5}$ \\ ${ }^{1}$ Department of Mathematical and Informatics Sciences, Physical Sciences and Earth Sciences of Messina University, Viale \\ Ferdinando Stagno D' Alcontres 31, Italy
}

${ }^{2}$ Le Studium, Loire Valley Institute for Advanced Studies, Orléans \& Tours, France

${ }^{3}$ Centre de Biophysique Moleculaire Laboratoire Interfaces, Confinement, Matériaux et Nanostructures (ICMN, France

${ }^{4}$ Istituto Nazionale di Alta Matematica "F. Severi" - INDAM - Gruppo Nazionale per la Fisica Matematica - GNFM

${ }^{5}$ CISFA “Interuniversity Consortium of Applied Physical Sciences" (Consorzio Interuniversitario di Scienze Fisiche, Italy

*Corresponding author: Emanuele Calabrò, Department of Mathematical and Informatics Sciences, Physical Sciences and Earth Sciences of Messina University, Italy

\begin{tabular}{lll}
\hline ARTICLE INFO & & ABSTRACT \\
\cline { 1 - 1 } Received: & & $\begin{array}{l}\text { Citation: Ectober 23, } 2020 \\
\text { Published: }\end{array}$ Fields in Membranele Calabrò, Salvatore Magazù. Applying Resonant Electromagnetic \\
& Sci \& Tech Res 31(4)-2020. BJSTR. MS.ID.005142.
\end{tabular}

\section{Mini Review}

The starting point of the results summarized in this brief note is represented by the assumption that electromagnetic fields (EMFs) in the microwaves (MWs) range can interact with the sophisticated electromagnetic circuits of livings, in particular at some frequencies named 'natural resonant frequencies' [1-5]. Resonance phenomenon occurs if a material is forced mechanically, or electrically, or acustically by an external forcing whose frequency is close to a natural frequency of the system, named natural resonance frequency. As a result, the oscillation of the system is amplified giving rise to very large vibrations that can destroy its structure. In the recent years some studies have shown that conventional methods for cancer treatment, in particular ionizing radiotherapy, often induce several bystander effects [6-16]. Taking a cue from this result, further researcher has been performed in order to investigate the effects of electromagnetic fields (EMFs) on patients affected by cancer. It resulted that the application of EMFs in cancer patients inhibited the disease progression prolonging the survival time of livings affected by cancer [17-22], even if these beneficial effects were not significant nor it was verified whether bystander effect was produced by this treatment.
Otherwise, resonance characteristic has led many scientists to hypothesize to use this phenomenon for inducing harmful effects on cancerous cells without damaging nearby healthy cells, avoiding bystander effect. To this aim, recent studies proposed a model to calculate natural resonant frequencies of cancer cells, so that an applied external EMFs working at one of such frequencies can interact with the system inducing resonance, damaging only cancer cells. Nevertheless, no experimental application of such theoretical models has been carried out up to now. Indeed, these models are based on civil engineering parameters like elasticity and tensegrity structures of a building in order to search different natural frequencies from that of an earthquake [23]. Starting from this point of view, other researchers assumed that the natural frequencies of cells would depend on mechanical properties such as elastic modulus, Young's modulus, density and shape of the cell, in order to search differences from natural frequencies of cancer cells $[24,25]$. Nevertheless, such models schematize cells by mechanical properties that cannot be considered representative in order to search natural frequencies of cancer cells, because cells are made up of billions of molecules each having its resonant frequency, so that a unique resonant frequency of a cell cannot be considered. 
Anyway, we should make a choice. We should state what physical chemical mechanism can be considered as the most important among cellular functions, in order to search a resonance between this mechanism and an applied EMF aimed to damage cancer cells. This mechanism is the ions flux across cellular membrane channels. Indeed, it have been demonstrated that such mechanism has a relevant role in cellular functions of cancer cells as it can regulate cancer's initiation, progression and proliferation [26-31]. Indeed, ions flux alteration in cells membrane channels is induced by pharmacological therapy such as chemotherapy or immunotherapy for cancer treatment. Nevertheless, significant bystander effects are induced by such treatments, as specified at the top of this note. However, it was shown that displacement of proteins' $\alpha$-helices in cells membrane channels are induced also by EMFs so that ions flux across cellular membrane channels change [32-34]. Hence, the authors of Ref.[32-34] have taken a cue from this result hypothesizing to emphasize this mechanism using EMFs at a frequency close to a natural resonant frequency of cellular membrane channel in cancer cells, inducing an amplification of change in ions flux across channels and a significant damaging of cancer cells. Otherwise, it was also demonstrated that there is a correlation between the displacement of cell channels $\alpha$-helices and the frequency of an applied high frequency EMFs confirming this scenario [35].

In addition, a significant inverse relation between the mitochondrial transmembrane potential and the $\alpha$-helices polarization was observed in SH-SY5Y neuroblastoma differentiated cells after exposure to a magnetic field associated to time exposure. Hence, another proof was provided represented by the result that an orientation of N-terminal helix in the Voltage Dependent Anion Channel (VDAC) is induced by a magnetic field even at low intensity, causing the channel to be prevalently in the open state. In addition, the mitochondrial transmembrane potential was observed to decrease because of decreasing in concentration gradient between outer and inner regions of mitochondrial membrane, following the Nernst-Planck equation [36]. This result also confirms the great dependence between EMFs and the displacement of proteins $\alpha$-helices forming the walls of cellular membrane channels, a phenomenon that should be amplified at a resonant frequency. Neither healthy neighboring cells would be damaged, as they should have natural resonant frequencies different from those of cancer cells, unlike what happens in other cancer treatments. In conclusion, the results obtained so far in this topic, that were briefly summarized here, should encourage to continue research in this direction, leading to think that this may be the future of cancer treatment, or at least an adjuvant treatment to traditional cancer therapies.

\section{References}

1. Edwards GS, Davis CC, Saffer JD, Swicord ML (1984) Resonant absorption of DNA molecules. Phys Rev Lett 53: 1284-1287.
2. Fröhlich H (1980) The biological effects of microwaves and related questions. Adv Electronics Phys 53: 85-152.

3. Grundler W, Keilman F (1983) Sharp resonances in yeast growth from non-thermal sensitivity to microwaves. Phys Rev Lett 51: 1214-1216.

4. Hyland GJ (1998) Non-thermal bioeffects induced by low intensity microwave irradiation of living systems. Eng Sci Educ J 7: 261-269.

5. Preto J, Pettini M (2013) Resonant long-range interactions between polar macromolecules. Phys Lett 377(8): 587-591.

6. Marín A, Martín M, Liñán O, Felipe Alvarenga, Mario López, et al. (2015) Bystander effects and radiotherapy. Rep Pract Oncol Radiother 20(1): 12-21.

7. Shemetun OV, Pilinska MA (2007) Radiation-induced "bystander" effect. Cytology and Genetics 41(4): 251-255.

8. Merle P, Morvan D, Caillaud D (2008) Chemotherapy-induced Bystander Effect in Response to Several Chloroethylnitrosoureas: An Origin Independent of DNA Damage? Anticancer Research 28(1): 21-27.

9. Chen HH, Jia RF, Yu L, Mei J Zhao, Chun L Shao, et al. (2008) Bystander Effects Induced by Continuous Low-Dose-Rate ${ }^{125}$. Seeds Potentiate the Killing Action of Irradiation on Human Lung Cancer Cells In Vitro. International Journal of Radiation Oncology Biology Physics 72(5): P1560-1566.

10. Mothersill C, Seymour C (2001) Radiation-Induced Bystander Effects: Past History and Future Directions. Radiation Research 155(6): 759 767.

11. Mukherjee S, Chakraborty A (2019) Radiation-induced bystander phenomenon: insight and implications in radiotherapy. International Journal of Radiation Biology 95(3): 243-263.

12. Taylor CW (2008) Breast cancer radiotherapy and heart disease. Oxford University, Trinity Term.

13. Pearson HES (1957) Coronary occlusion following thoracic radiotherapy, 2 cases. Proc R Soc Med 50: 516.

14. Pearson HES (1958) Incidental dangers of x-ray therapy. Lancet 1: 222 223.

15. Cuzick J, H Stewart, L Rutqvist, J Houghton, R Edwards, et al. (1994) Cause-specific mortality in long-term survivors of breast cancer who participated in trials of radiotherapy. J Clin Oncol 12(3): 447-453.

16. Darby SC, Marianne Ewertz, Paul Mc Gale, Anna M Bennet, Ulla Blom Goldman, et al. (2013) Risk of Ischemic Heart Disease in Women after Radiotherapy for Breast Cancer, The New England Journal of Medicine 368(11): 987-998.

17. Barbault A, Costa FP, Bottger B, Reginald F Munden, Fin Bomholt, et al. (2009) Amplitude-modulated electromagnetic fields for the treatment of cancer: Discovery of tumour-specific frequencies and assessment of a novel therapeutic approach. J Exp Clin Cancer Res 28: 51.

18. Costa FP, A C de Oliveira, R Meirelles, M C C Machado, T Zanesco, et al. (2011) Treatment of advanced hepatocellular carcinoma with very low levels of amplitude-modulated electromagnetic fields. Br J Cancer 105(5): 640-648

19. Orel VE, Yu I Kudryavets, S Satz, N A Bezdenezhnih, M I Danko, et al. (2004) Effects of mechanochemically activated doxorubicin and $40 \mathrm{MHz}$ frequency irradiation on humanA-549 lung carcinoma cells. Exp Oncol 26(4): 271-277.

20. Kirson ED, Gurvich Z, Schneiderman R, et al. (2004) Disruption of cancer cell replication by alternating electric fields. Cancer Res 64(9): 32883295 .

21. Kirson ED, Gurvich Z, Schneiderman R, Josef Vymazal, Jean F Soustiel, et al. (2007) Alternating electric fields arrest cell proliferation in animal tumour models and human brain tumour. Proc Natl Acad Sci USA 104: 10152-10157. 
22. Sersa G, Cemazar M, Miklavcic D (2003) Tumor blood flow modifying effects of electrochemotherapy: A potential vascular targeted mechanism. Radiol Oncol 37(1): 43-48.

23. Bhimarao M (2011) Collapsing Cancer Cells: Exploiting the Elasticity and Natural Frequency of a Cancer Cell's Cytoskeleton. Project n.S1703: California State Science Fair.

24. Ronchetto F Barone D, Cintorino M (2016) Natural frequency of cancer cells as a starting point in cancer treatment. Curr Sci 110(9): 1828-1832.

25. Wee H, Voloshin A (2012) Modal Analysis of a Spreading Osteoblast Cell in Culturing. 38th Annual Northeast Bioengineering Conference (NEBEC 2012), 16-18 March 2012. Philadelphia, Pennsylvania: USA.

26. Becchetti A (2011) Ion channels and transporters in cancer. 1. Ion channels and cell proliferation in cancer. Am J Physiol Cell Physiol 301(2): C255-C265.

27. Hanahan D, Weinberg RA (2000) The hallmarks of cancer. Cell 100(1): 57-70.

28. Lang F, Stournaras C (2014) Ion channels in cancer: Future perspectives and clinical potential. Phil Trans R Soc B 369(1638): 20130108.

29. Oosterwijk E, Gillies RJ (2014) Targeting ion transport in cancer. Phil Trans R Soc B 369(1638): 20130107.

30. Peruzzo R, Biasutto L, Szabò I, Leanza L (2016) Impact of intracellular ion channels on cancer development and progression. Eur Biophys J 45(7): 685-707.

ISSN: 2574-1241

DOI: $10.26717 /$ BJSTR.2020.31.005142

Emanuele Calabrò. Biomed J Sci \& Tech Res

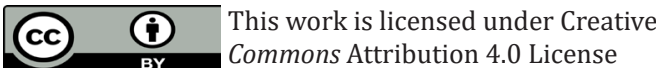

Submission Link: https://biomedres.us/submit-manuscript.php
31. Turner KL, Sontheimer H (2014) Cl2 and Kp channels and their role in primary brain tumour biology. Phil Trans R Soc B 369(1638): 20130095.

32. Calabrò E, Magazù S (2017) The $\alpha$-Helix Alignment of Proteins in Water Solution towards a High Frequency Electromagnetic Field: a FTIR Spectroscopy Study, Electromagnetic Biology and Medicine 36(3): 279288.

33. Calabrò E, Magazù S (2018) Resonant interaction between electromagnetic fields and proteins: A possible starting point for the treatment of cancer. Electromagnetic Biology and Medicine 37(3): 155168 .

34. Calabrò E, Magazù S (2019) New Perspectives in the Treatment of Tumor Cells by Electromagnetic Radiation at Resonance Frequencies in Cellular Membrane Channels. The Open Biotechnology Journal 13: 105-110.

35. Calabrò E, Magazù S (2020) Correlation between Cell Channels $\alpha$-Helices Displacement and Frequency of Applied Electromagnetic Field. International Journal of Pharmaceutical and Phytopharmacological Research (eIJPPR) 10(1): 1-7.

36. Calabrò E, Magazù S, Currò M, Ientile R (2020) The inverse relation between mitochondrial transmembrane potential and proteins $\alpha$-helix in neuronal-like cells under static magnetic field and the role of VDAC. Electromagnetic Biology and Medicine 39(2):176-182. 